\title{
Interactive Hull Form Transformations using Curve Network Deformation
}

\author{
Marcus Bole, PolyCAD, Gosport/UK, marcus.bole@polycad.co.uk
}

\begin{abstract}
All hull surface transformation techniques used today are applied to the space in which the surface definition resides, deforming it geometrically. This approach is usually successful for changes to large portions of the surface but often smaller features can be harder to change, becoming undesirably distorted in the process. If a transformation toolkit within a design tool is unable to accommodate a particular feature, the change must be made by hand and is likely to take significantly longer than if a transformation had functioned effectively.

For a hull surface defined using a curve network, local features can be identified by reviewing the hull form topology, Bole (2006). However, rather than invoke a spatial deformation specifically designed for each feature, the curve network can be used to transmit changes across edges and faces, adapting to any features that may be present. This paper presents an approach to hull surface transformation based on identifying features in the form topology and allows changes using both interactive mouse manipulation and numerical parameters. A rich transformation tool kit is created which allows the user to confidently make rapid hull changes in conjunction with existing surface definition techniques.
\end{abstract}

\section{Introduction}

The process of developing a new hull form can be costly and time consuming. The qualities of a hull form, developed without reference to a previous design are unknown. Designs with no heritage often require extensive analysis to verify that they perform as desired. The preferred alternative to this approach is to take an existing design and modify it to suit the new requirements of the project. The performance characteristics of a derived design should be similar to the original as long as the changes between the two hull forms remain conservative.

To change hull form shape many commercial hull form design software include the ability to transform the hull surface. The most prolific approach is based on the methods documented by Lackenby (1950) and allow the designer to adjust the hydrostatic characteristics of a hull form by either changing the length of the parallel middle body or distorting the entrance and run. Simpler changes using combinations of scaling and translation can be implemented providing geosim changes. In both cases, the changes made by these techniques cover significant areas of the hull surface. For minor changes, it's necessary for the surface to be changed by directly modifying the definition.

The nature of the changes to the hull form and any tidying up that must take place after the surface has been changed means that the activity can only be undertaken by the person in the organization responsible for the hull surface, having been trained in the skills required to make changes reliably. When a project is innovative or resources must be efficiently managed, other members of the project may need to use a representative hull surface to check design or production issues. However, often the project member responsible for hull surface modifications is unavailable or a design does not exist. In these cases, a user-friendly design tool is required to allow a range of changes to be made quickly and easily to a hull surface without requiring the full range of skills required for detailed hull surface design activities.

\section{Some Notable Hull Transformation Techniques}

Before computers were introduced into design offices, hull surfaces were manipulated on the drawing 
board by hand. This process was incredibly time consuming because the designer was responsible for ensuring that the $2 \mathrm{D}$ views would produce a viable 3D surface. Any hull transformation techniques used in the design process needed to avoid introducing unnecessary manipulation and complex calculations. Lackenby documented two practical techniques generally known as "1-Cp" and "Lackenby" which change a hull form by longitudinally translating hull sections using calculations that could be made by hand. Both techniques use relatively simple scaling functions to deform forward and aft regions of the hull surface. In addition, Lackenby developed functions which would convert the desired hydrostatic changes into the amount of adjustment that should be applied to the hull form using the transformation functions. Lackenby's methods are still used in modern hull design applications but it is necessary to extend the technique to deal with modern surface representations and distortion beyond the limits of the section area curve.

Once computers began to be used to manipulate hull surfaces richer techniques for transforming the hull form were developed. Söding (1977) aimed to provide a generic solution to hull form transformation. The transformation defines a mapping from the original to the derived surface, with the mapping consisting of a structure of functions in each coordinate component. The functions themselves may be stored in a library, accessed when a change is required or defined by the user. The solution was developed in association with design software because structure used to formulate and apply the change is an important aspect of this solution, using specific command syntaxes to invoke and specify mathematical changes. The approach provides a rich and open set of tools for changing hull shape and it seems possible for the user to develop their own custom transformation rules. However, this technique was developed prior to the introduction of standardised user interfaces, like Windows, and it would have been very difficult for practical designers, who often show impatience with complex tools, to get the best from the system.

Free form deformation (FFD) is a recent introduction that has its origins in the 3D graphical design software. The principle behind FFD is to enclose a complex geometric shape within a simpler shape which under goes distortion. The distortion of the simpler shape provides a mapping to change the coordinate positions of the more complex shape. A simplistic example is to use a cuboid that when distorted regularly can cause contained geometry to be translated and scaled. If individual corners of the cuboid are translated, the contained geometry can be twisted and sheared. In more complex examples, the enclosing volume may be represented by trivariate NURBS volumes allowing highly complex and non-linear distortions. As the enclosing volume is geometric, it is very easy to control using interactive or parametric means and is one of the reasons why it has become popular. However, while the technique is very successful at making informal changes to geometry, a requirement of hull design is the requirement to make precise changes to the surface. This can be achieved with the FFD technique but it is necessary to prepare the deforming volume so that its changes will be compatible with the structure of the hull surface and respect its features. This takes time and means that software implementations using this approach can only provide a limited range of hull form changes if customisation is to be avoided.

\subsection{The Limitations of Geometric Hull Form Transformation}

Hull forms, particular of commercial ships, have specific features which contribute to the performance of the vessel. These features have limitations on the way that they can be distorted otherwise they may have a detrimental effect on performance. Each of the techniques discussed here change the hull form by defining an explicit mathematical set of transformation rules which are applied to the geometric definition of the hull surface. In order to avoid undesirable distortion to hull surface features, the transformation must account for the features by coding into the technique. In the case of Söding's approach, the transformation functions must be designed to fit to the features of a hull surface. In FFD, the initial distorting geometry may have to be tuned to fit the surface. Lackenby's techniques requires the locations of the parallel middle body to be established prior to applying the transformation to the hull offsets and it does not make any specific allowance for the shape of the hull form beyond the limits of the section area curve. These limitations make it difficult to incorporate a wide range of efficient generic transformation tools into hull design software and it often results in the 
designer becoming frustrated when it does not work correctly or choosing to modify the surface by directly manipulating the definition.

\section{Modern Hull Form Representation Techniques with Respect to Transformation}

The offset data of Lackenby's era is rarely used in modern shipbuilding, with almost all tools using mathematical surfaces, particular NURBS, due to the wide number of applications these representations support. NURBS, in particular, have become ubiquitous because complex shapes can be accurately represented by a very small amount of structured definition data. Consequently, it's very easy to transfer surface representations between software tools and allows a single definition to be used for applications as far ranging as detailed hydrodynamic analysis to numerically controlled fabrication. Furthermore, the definition used to control NURBS surface is a grid of coordinate points, which when presented to the user within a design tool, can be manipulated to change shape with ease. The control points can also be manipulated using coordinate transformations without any significant complexity.

While the introduction of NURBS enabled sharing of the hull representation and made design of individual surfaces easier, it still requires a lot of skill to produce a surface of the quality required for efficient hydrodynamic and production performance. In particular, it is difficult to fit the rectangular shape of these surfaces to the varied shapes which can be found in hull forms. A natural extension is to allow the use a number of surface patches to represent the hull form but this introduces the problem of ensuring smooth continuity between surface patches. There are various techniques for achieving this, but the most appropriate for ship hull forms is to use a network of curves, from which a patchwork of surfaces is generated. This approach, arguably, requires more skill than individual patches to generate a good quality hull form but it does allow the designer to work with curves, which often offer a better technique for describing surface shapes than directly manipulating the surface itself. Consequently, this technique will often be used produce or change complex hull surface in a much shorter time than direct manipulation of the surface definition. As the curves are defined by coordinate points, they can be manipulated by coordinate transformations much like the surface definition. However, topology is often used in the curve definition to allow curves to reference others and non-uniform transformation techniques must take extra care to avoid changing the curve network in such a way that references become invalid preventing the hull surface from being generated.

\section{IntelliHull and the Introduction of Form Topology}

While in a role that involve the regular definition of hull surfaces, the author noted that almost every hull surface uses a similar structure of definition, yet it was necessary define a new definition structure each time a different surface was required. Furthermore, the structure could easily be sketched and annotated to describe how different shape characteristic would be distributed about the surface, Figure 1.
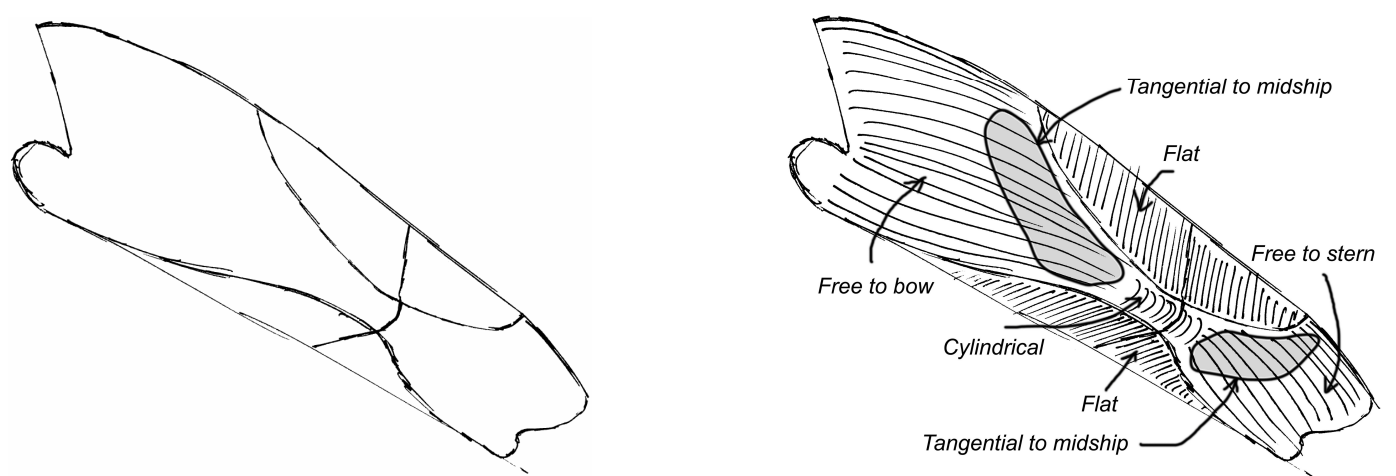

Fig.1: An outline sketch of a hull form showing typical boundary and feature curves (Form Topology) (left), separating the regions of different shape characteristics (right). 
IntelliHull, Bole (2006), was an attempt to use this structure to generate a hull form surface producing a tool which combined the ideas of both parametric hull generation and traditional hull surface manipulation to allow the designer to manipulate shape interactively and control dimensions and hydrostatic properties numerically. The concept of Form Topology was introduced to allow the software to understand the designer's definition by representing the structure and relationships between the different features and shapes that can be found in a hull form surface definition. Graphically, Form Topology has the same shape as the sketch, Figure 1, but may be represented using a topological graph data structure and curve geometry, (Figure 5). Using Form Topology, IntelliHull software can identify features such as the Parallel Middle Body or the ends of the ship, creating flat or curved surface definition in those locations respectively. Form Topology could also be used to expose form parameters measured from key features in the designer's definition or the generated hull surface. Modification of these parameters invoked a specific transformation to change the feature guided by the Form Topology.

IntelliHull was limited to producing a single surface using a lofting technique across a transverse set of curves. As a design progressed, it became increasingly difficult to introduce smaller features because the surface definition was restricted to a maximum number of definition curves each with a consistent number of control points. Experience with commercial tools highlighted that the only way to extend the Form Topology concept beyond the generation of a single hull surface was to use a curve network to generate more flexible representations and that the technique would function better if focused on transforming the hull surface definition rather than on the hull form generation problem.

\section{Hull Surface Definition Using Curve Network Topology and Hierarchy}

A curve network allows the designer to build up a surface definition in stages, unlike direct surface manipulation where the designer starts with a complete surface and must deform it into the desired shape. In the initial stages of the process, Figure 2, key shape curves, such as the midship section and the boundaries of the surface definition will be defined, such as the centreline and deck. Subsequently, curves will be added between key curves to refine the shape. Some surface design tools allow curves to be attached to previously defined curves, creating a hierarchy. Consequently, curves lower down the hierarchy will update as result of changes to curves higher up. This feature is useful in the early stages of defining a surface as it may only necessary to accurately position the initial key curves, as subsequent curves may reference them without the need to position control points explicitly.
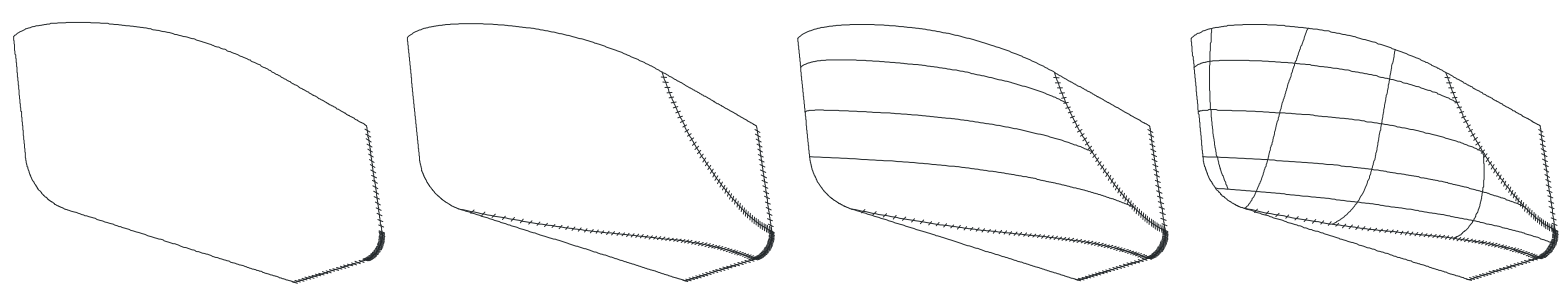

Fig.2: Create a simple bow of hull, beginning with the boundaries, features followed by waterlines and sections. Curve created at each stage actively reference curves generated in previous stages.

In addition to control points, rules may be assigned to components in the curve network to control or constrain the shape of curves without the designer having to specifically position the control points. These attributes can be summarised as follows:

Point attributes: These attributes can be assigned to control points in the curve to change its shape as it passes through the point. Usually, this will involve constraining the tangent of the curve or introducing a knuckle, Figure 3. Rules assigned to point pairs can introduce straight segments or blends in the curve shape. 




Fig.3: Point attributes, beginning with the raw B-Spline curve, with a straight, knuckle and blend applied respectively.

Curve Attributes: Curve attributes can be used to introduce the same constraints as point attributes, but assign the effect to the referencing points of any attached curves. The result is that the attribute affects all attached points without having to assign an attribute to each point individually. Shapes such as knuckles and surface angles can be generated in the surface using this method, Figure 4. Curve attributes can generate tangents in referencing curves based on the definition of the surface attribute. In the area of the parallel middle body these attributes can be used to ensure that curves enter the flat of side, bilge radius and flat of bottom with the correct tangent.
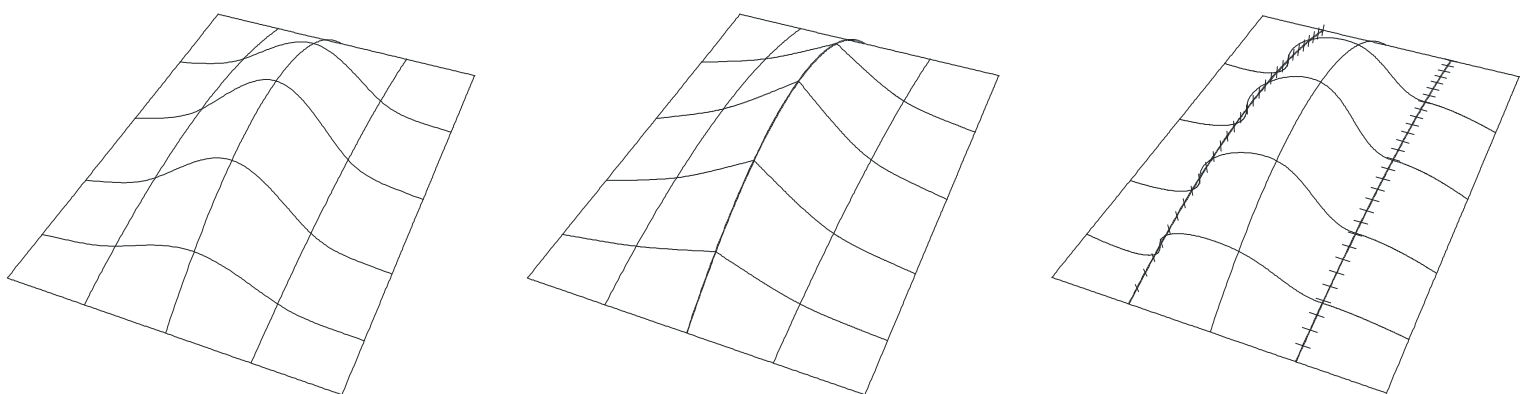

Fig.4: Curve attributes, beginning with the raw curve network and then showing the effects of a knuckle attribute or the application of curve tangent constraints.

Surface Attributes: These attributes are used to simplify curve definition by locating it within a surface effectively making it two dimensional. This makes manipulation and management of certain key curves such as midship sections and flat of side/bottom curves easier. As mentioned above, surface attributes can be used in conjunction with curve attributes to constrain tangent shapes at points. The implementation used in this study restricts surface attributes to planar constraints, although more elaborate definitions are available in other design tools.

The ability to add attributes to control curves, and hence surface shape, is an advantage that other definition techniques which are purely geometrical do not have. However, care must be taken with attributes when it comes to transforming the surface. It is possible to introduce attributes which cannot be transformed geometrically. In particular, it is hard to transform an attribute which introduces a tangent angle into a curve using a non-uniform coordinate transformation. In the curve network implementation used in this study, the designer can only assign attributes that can be safely transformed. Furthermore, none of the attributes implemented have any extra parametric information beyond that of the curve control points because this could introduce the need for specific transformation rules to change the attribute as well as the curve geometry.

\section{Identifying the Form Topology in the Curve Network}

As mentioned in the previous chapter, the procedure used to create a curve network has a very natural feel with respect to the design process, with curves representing key features of the surface being defined before any free form surface shape is defined. The explicit relationships between curves for certain types of hull form allow properties which may be common to all surface definitions of that type to be identified. For example, for traditional monohull ship hull forms:

- The midship section curve will lie within the transverse plane and, in a hull form with parallel 
middle body, the hull surface will be normal to the plane in which the curve is defined. The midship section curve will lie approximately midway along the length of the hull form.

- Tangents of curves at points attached to Flat of side and Flat of bottom curves will be tangential to the parallel middle body.

- Within the parallel middle body region of the flat of side and flat of bottom, there will be no need for definition curves.

- Etc.

Furthermore, certain curve relationships will be known. For example, the flat of side and flat of bottom curves will attach to the midship section curves forming the extents of the parallel middle body. This relationship can be used to establish the portion of the midship section curve that defines the bilge radius. By continuing to extend these heuristic rules to the other key features of the hull form, the network of Form Topology curves may be established by checking the location, connections and attributes associated with each element within the wider curve network. Once the Form Topology curve network has been found, Figure 5, form parameters in addition to the main dimensions and hydrostatic properties may be identified. The free form regions of the hull form, in the entrance and in the run, which would need to be adjusted to change the volumetric properties of the hull form, are also identified, highlighted later in Figure 10. Consequently, the approach used in IntelliHull, to measure parameters from the surface definition and invoke a transformation when changed can be used as an additional tool alongside the manual manipulation of the curve network definition.
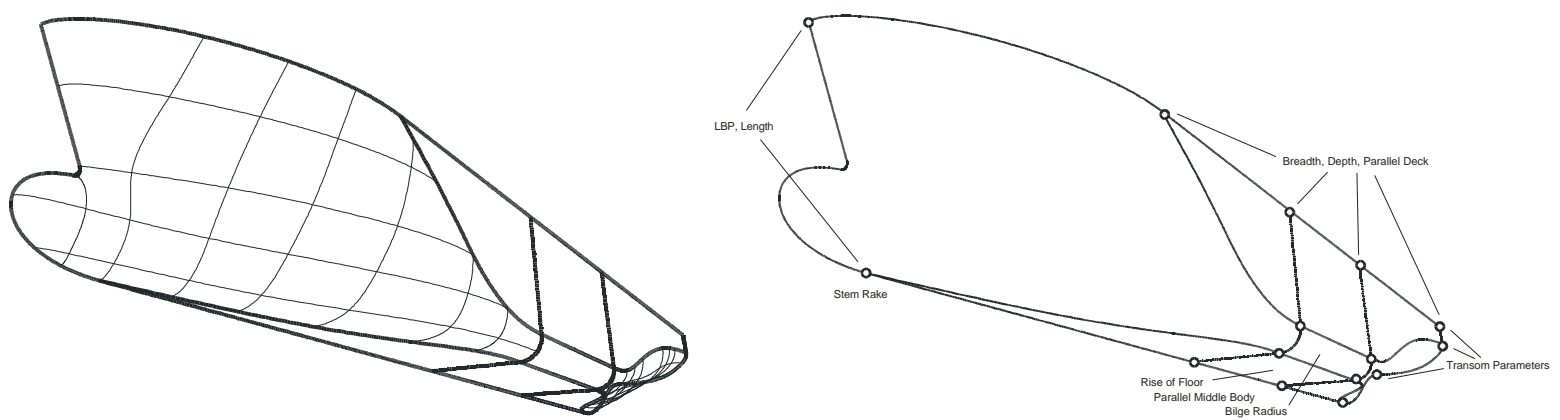

Fig.5: The Form Topology formally identified in the curve network (left). Design parameters can be identified in the Form Topology once the individual curves within the structure are identified (right).

\section{Using Topology and Hierarchy to Transform a Hull Form}

\subsection{Transformation guided by the Curve Network Hierarchy}

As the curves representing the key features of the hull form are usually designed first, appearing at the top of the curve network hierarchy, it is natural to assume that this structure can be used to deform the hull surface definition. By modifying key curves, any descendant curves, attached through referenced points will update to the changes. Such a technique, Sticky Splines, van Overveld (1996), has been explored for use with 2D animation. Unlike the curve network used to define a hull form, it consists entirely of free form splines and doesn't necessarily use the additional attributes. The use of curve attributes makes the process of transforming the network a little easier. In the case of Sticky Splines, the technique has to identify any specific features from the arrangements of control points and introduce constraints to ensure that the correct transformation is applied. A process to identify special features and introduce particular transformations into the curve network is not necessary if attributes are present, and consequently, the attribute itself will take care of any specific updates. Consequently, all that is necessary is to find a method of transforming free control points that lie on curve segments between the constrained control points that are attached to other curves or control points representing discontinuities in the curve, defined using attributes. A simple transformation rule was developed, Figure 6, inspired by the Elastic option available when modifying Spline curves in CorelDraw, a vector graphics drawing software application. 


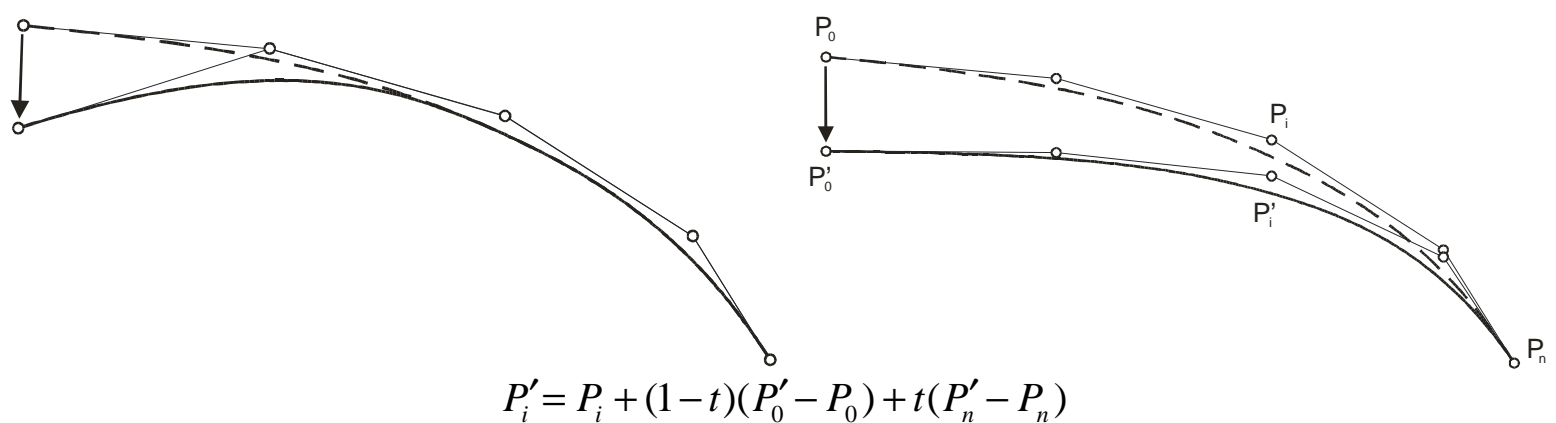

Where $\mathrm{t}$ is the normalised (chord) distance along the curve from $\mathrm{P}_{0}$ to $\mathrm{P}_{\mathrm{n}}$

Fig.6: Translations of curve end or attachment points need to be distributed to all internal free points on the curves to achieve the full transformation

The simplicity of this approach was really attractive but it was found to have limitations because the order in which the transformation was applied was entirely governed by the user. In vessels, without flat of side/bottom, particularly small craft and yachts, the natural order in which the curve network hierarchy is composed, for the ease of design, is not necessarily appropriate for the best transformations, Figure 7. Hence the designer is forced to use an undesirable hierarchy to utilise these transformations properly.


Fig.7: Deformation of a yacht hull form using the curve network hierarchy. In this example, the transformation is successful (centre) if waterlines F, E, D are connected to sections A, B and C, but not if the relationships are reversed (right).

However, while this approach was found to be unsuitable for formal hull form transformation it was found to be very useful when creating the hull form because descendant curves update as a result of changes further up the hierarchy. This means that the surface definition subtly maintains its general style and fairness. In fact, the user is generally not aware of this process happening until the feature is switched off.

\subsection{Transformation guided by Form Topology Curve Network}

The approach explored in the previous section performed poorly because it relied on the user to provide the structure of the transformation. Yet, if the correct structure was provided, the transformations performed remarkably well. As the structure of the users curve network cannot be relied upon, a solution is to generate a separate hierarchy based on the users curve network and this can be found by extracting the Form Topology curve network from the hull form definition and treating it as a unidirectional graph. Consequently, the issue of the order in which curves are connected is avoided. Based on the elements of the Form Topology graph data structure, i.e. the vertices, edges and faces, a new transformation order can be devised as follows.

1. Translate selected vertices in the Form Topology.

2. Update any planar constraints associated with the selected vertices.

3. Distribute the transformation around the Form Topology graph using a Breadth First Search, as described in 7.2.1.

4. Deform the edges of the Form Topology graph by calculating new positions of free control 
points on curves associated with each edge, as described in 7.2.2.

5. Translate all curve control point positions associated with each face in the Form Topology using the difference between two multi-sided surfaces blended using the face edge geometry, as described in 7.2.3.

6. Assign each new position to the control points of the curves (in the order of the users curve network hierarchy).

It is not necessary to identify the role of each curve in the Form Topology curve network graph at this stage, it is only necessary to identify whether a curve forms part of the Form Topology. This can be achieved by selecting all the curves which either form the surface boundary or have curve attributes. It may be necessary to do a deeper investigation to identify cases where the user has used many individual point attributes instead of a single curve attribute or the Form Topology introduces a curve which is important to the design process by does not need use a attribute to generate a specific surface feature, such as a midship section on a hull form with no parallel middle body features.

\subsubsection{Transformation of Form Topology Graph Vertices}

The vertices of the Form Topology curve network occur at the intersections of key curves. In most cases, specific hull form design parameters can be associated with individual or pairs of these vertices. A transformation can be instigated by translating the vertex and subsequently distributing the change throughout the Form Topology curve network graph.

As the position of vertices corresponds to a control point on a curve in the Curve Network, it must respect any attributes which constrain its position. As vertices lie at the intersection of a number of curves, its position may be constrained by a number of planes as defined by any surface attributes. As planes can generate a significant amount of constraint, the Form Topology curve network is analysed to identify any planes attributes and any faces with planar geometry within the Form Topology graph. Planes associated with the initially translated vertices need to be repositioned first with respect to the applied changed because the update of the remaining vertices will respect these planes when the transformation is distributed. Once any relevant planes have been updated, the remaining vertices respect planar constraints as follows:

- Vertices associated with a single plane can only be moved by the translation component parallel to the plane.

- Vertices associated with two planes are constrained to the line defined by the intersection of the two planes and can only be moved by the translation component parallel to the line.

- Vertices associated with three or more planes must lie at the intersection of the planes and cannot be moved.

Once an initial vertex has been selected for change, it must pass the translation on to neighbouring vertices through adjacent edges. The translation may be affected any attributes the curve geometry associated with the edge. Three different types of edge geometry can be identified and a rule for each case can be defined controlling how a translation at a source vertex may affect a destination vertex at the other end of the edge:

- Free Form edges have one or more free control points associated with the curve geometry representing the edge. As this edge is free to move about under the user control, the translation applied to a vertex at one end of the edge is not transferred to the adjacent vertex.

- Edges representing straight lines have no free control points. Consequently, only the translation component normal to the line is passed on to the adjacent vertex allowing the edge to change length and position but not direction.

- Edges representing blends have no free control points but are shaped by attributes affecting the curve tangents present at the terminating vertices. A number of rules are possible, but to ensure the overall size is maintained, the translation is transferred along the edge without 
modification.

When a translation is being distributed around the Form Topology graph, a vertex could potentially receive a translation from a more than one neighbouring vertex. This means that the distribution must be carefully done to make sure the translation is passed around in a synchronised manner, this is achieved using a breadth first search. The following procedure is used.

1. The initial selected vertices are translated and are collected into an input array.

2. Each vertex in the input array passes its translation on associated edges which have adjacent vertices that have not been translated. The edge modifies the translation based on the edge geometry rules, stores the result and collects the adjacent vertex into an output array. The vertex is marked as transformed.

3. Each vertex in the output array checks its associated edges for translations, and calculates the average translation. The translation is constrained to any planes associated with the vertex.

4. The output array becomes the input array and the procedure returns to step 2 until all vertices have been processed.

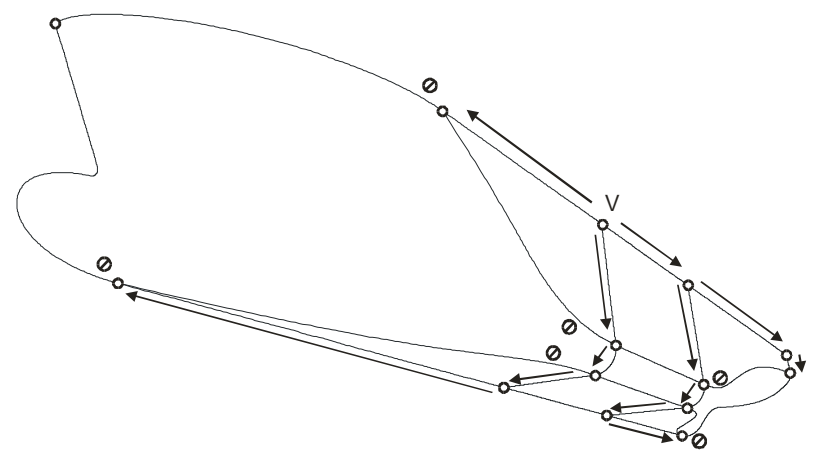

Fig.8: Translation of vertex ' $\mathrm{V}$ ' is distributed along the edges using a breadth first search. The translation is adjusted based on the geometrical definition of the underlying curve. In the case of freeform edges, further distribution of the translation is terminated.

\subsubsection{Transformation of Free Control Points associated with Form Topology Graph Edges}

After the vertices have been processed, the new position of free control points associated with each edge is calculated using the technique described in 7.1 for curve segments.

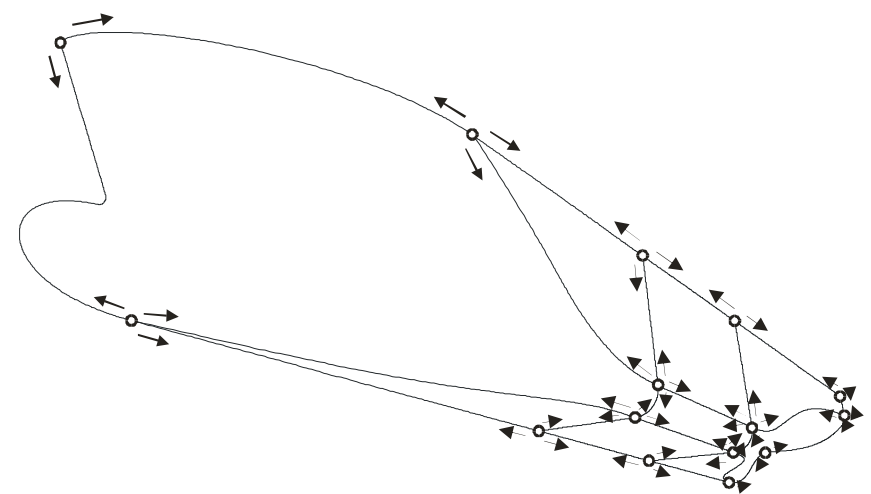

Fig.9: Vertex translation is subsequently transferred to the curve geometry associated with each adjacent edge.

\subsubsection{Transformation of Free Control Points associated with Form Topology Graph Faces.}

Although it would be possible to update each curve segment associated with a Form Topology graph face using the process described in 7.1, the curve itself would only change based on the translations occurring at the points where curve connects with the edges of the face. Consequently, it could only 
respond to changes face edges to which it is not directly attached by receiving changes from the curves it references. We have already established that this is not a reliable method of transformation. What is required is to extend the concept that updates curve geometry associated with an edge with respect to its bounding vertices, to updating curve geometry on a face with respect to its bounding edges. This can be achieved by using a surface defined across the face and generating two surfaces, one representing the initial state of the Form Topology and one the transformed Form Topology after vertices and edges have been updated. By projecting each free control point onto the surface and subsequently identifying the parametric location the point, the translation applied to the free control point can be determined by the change of position of the projected pointed as it moves to the transformed surface at the same parametric location. As the free form faces of the Form Topology curve network are unlikely to be four sided, it's necessary to blend a multi-sided surface, in this case a Coons patch using Gregory's method, Gregory (1982), across the face.

This transformation may be extended by using an additional parameter to bulge or flatten the surface and can be used to control the volumetric properties of a hull form independently of changes experienced through the face edges. This is discussed more in 8.2.
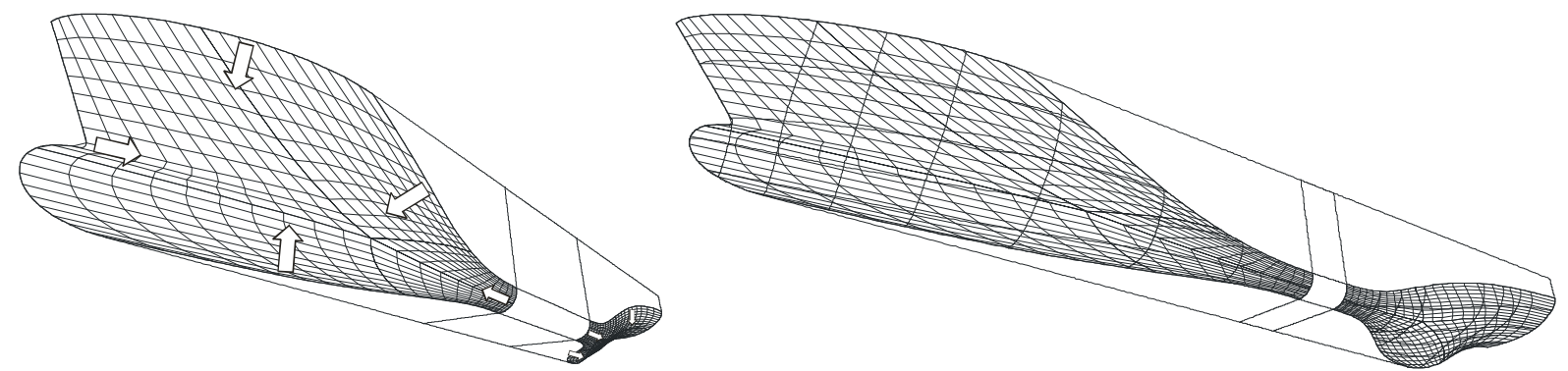

Fig.10: Edge deformation is transferred into a multisided surface blended across the faces, (left). The difference in the surface in the pre- and post-transformed form topology curve network is used to deform curve geometry associated with the face (right).

\subsubsection{Reconnection of Curves in the Curve Network}

As the Form Topology curve network is deformed, it may not be possible for certain curves to continue to be connect to referenced curves, for example, if the curve plane no longer intersects with the referenced curve or the update causes curves to overlap, invalidating the curve network topology and preventing the surface patches from been generated correctly. Consequently, once the Form Topology curve network is deformed a reconnection process needs to be applied to validate correct connections. At the time of writing a process for achieving this has been proposed but not yet investigated.

\subsubsection{Parametric Transformation of Linear Dimensions}

Once curves in the Form Topology are tagged, vertices associated with specific parameters can be found by searching at the intersection of key curves. For example, the forward end of the deck may be found by looking for the intersection of the flat of side curve with deck boundary of the surface. The values of design parameter can be then be established by measuring the either the absolute position of Form Topology graph vertices or relative positions between a number of vertices. Changes to parametric values can then be applied as vertex translations. In some cases, it may be possible to directly assign the change in value to one of the coordinate components of the translation. For example, to increase the breadth of a vessel, the increase could be assigned as a transverse translation of all vertices associated with the parallel middle body, Figure 11. In other cases, it may be necessary to devise an algorithm to distribute the change across a number of vertices. For example, in changing the bilge radius, it will be necessary to calculate a variety of different translations to reposition the vertices terminating the bilge radius arc moving their position along the flat of side and flat of bottom planes, accounting for rise of floor etc, if present. 


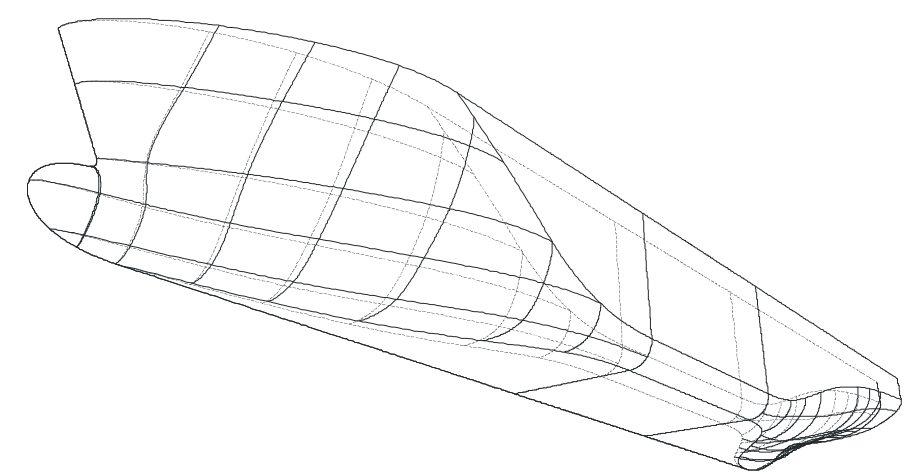

Fig.11: The curve network resulting from a change in breadth applied to vertices within the parallel middle body at the deck.

\subsubsection{Interactive Transformation}

The vertices of the Form Topology Curve Network may also be manipulated using the mouse providing another way of interacting with the definition in addition to directly manipulating the free control points on each curve. However, considering the amount of processing required to present the transformation interactively and the fact that the Form Topology Curve Network quickly becomes badly deformed when indiscriminate changes are made, its often better to restrict changes to the subtle transformations that can be achieve using parameters.

\section{Using the Form Topology Curve Network to make Hydrostatic Transformations}

The Form Topology curve network offers some advantages over the techniques discussed in the introduction. By breaking down the surface definition into several regions, we can identify the areas that can be deformed to change the volumetric properties, such as the free form faces in the entrance and the run and those that cannot be transformed, such as those in the parallel middle body. Furthermore, if the available adjustment within individual regions is not sufficient, we may look at more extensive deformation of the Form Topology curve network in order to achieve the hydrostatic parameters that the designer has in mind. The technique may also provide the designer with a number of different deformation options with which to achieve the change.

Fundamentally, the Form Topology curve network, with respect to ship hull forms, offers two main techniques for adjusting the hydrostatic properties of the hull form, similar to the approaches described by Lackenby:

- The extents of the parallel middle body may be adjusted in a manner similar to 1-Cp and may be achieved through the Form Topology curve network by moving the vertices on the midship section curves at either end of the parallel middle body (PMB) longitudinally. Unlike, the 1$\mathrm{Cp}$ method, the deformation does not have to be applied uniformly across the entrance or run. If no further vertices are moved, the change is restricted to the movement of the midship section curves only and no deformation takes place at the ends of the vessel or to deck shape. Further rules may be introduced which translate the position where the flat of side meets the deck, either by the same amount that the midship section is displaced or by maintaining the ratio of the position of this point between the end of the vessel and the parallel middle body.

- The free form faces of the Form Topology curve network in the entrance and the run may be adjusted. The deformation only affects the curves crossing the free form face region so that, unlike the Lackenby method, there is no deformation in the deck, flat of side or flat of bottom. This approach offers a much more subtle change than Lackenby's method.

These two approaches may be combined together when making more extensive changes to the hull 
form.

\subsection{The Architecture of Iterative Hydrostatic Transformations}

The techniques documented by Lackenby are restricted to uniform longitudinal translations using simple functions without discontinuities and were designed to be applied to hull offsets. Consequently, it was possible to develop an analytical model to guide the magnitude of the deformation to achieve the desired set of volumetric properties without too much difficulty. However, when these transformations are applied to modern hull surface representations the analytical models of the transformations are not as accurate as it is necessary to use a number of iterations to achieve the desired volumetric properties to a desirable degree of accuracy.

Care must be taken when using iterative techniques because each iteration is often expensive to compute. With respect to the Form Topology curve network, the computing expense associated with each iteration is significant as it is necessary to compute the surface resulting from the curve network, the Form Topology curve network and integrate over the surface to identify the volumetric properties. A significant amount of effort was given to optimising the processing that occurs during each update, although it was found that the surface integration was the most expensive element. As each iteration is expensive, the number of iterations must be minimised. This can be achieved by using the following recommendations:

\subsubsection{Explore the limits of the Deformation prior to iterating}

Bracketing is a very effecting way of controlling iterative techniques constraining an iterative search to a closed parameter space. As well as creating a very efficient way of managing the algorithm so that it arrives at a solution, it also allows the algorithm to identify if the requested solution is outside the parameter space and terminate further iterations. However, efficient search algorithms are often generic and it is often not possible to tailor them to the specific issues associated with the application. The process of identifying the brackets needs to both decide on the appropriate range of deformation and can check that the designer has requested a solution that can be found in the bracketed parameter space.

In order to identify the range of deformation associated with face in the Form Topology Graph, an iterative technique is used to deform curves to identify the point when undesirable shape begins to appear. This process itself must be bracketed. In this case, the brackets are set to be artificially large and the definition curve algorithms are robust enough to deal with significant deformation beyond what would be deemed suitable for a hull form. Subsequently, hydrostatics obtained when the hull surface is generated at the minimum and maximum range of deformation identify the range of change available in the displacement and longitudinal centre of buoyancy (LCB).

\subsubsection{Develop a Model to Guide or Simplify Iterations}

When controlling displacement and LCB, two control parameters are required in the entrance and run to change hull shape which suggests the use of multi-parameter iteration algorithms. However, this can be avoided by introducing an empirical model which translates displacement and LCB into two independent parameters. IntelliHull introduced a simple model which achieved this, although the approach used by Lackenby's is more advanced converting displacement and LCB into values of prismatic coefficient in the entrance and run. The introduction of this technique reduces the number of iterations required and simplifies the iteration algorithm implementation. It also means that numerical quantities used in the software remain meaningful to the problem. Before these models can be used, they often need to be initialised with hydrostatic properties from the hull form. The method introduced in IntelliHull is initialised using the information from the bracketing process described previously.

\subsection{Extending the control of Surfaces blended across faces of the Form Topology Graph}


The iterative process used to change the shape of the hull surface requires the use of two control parameters which will be adjusted, deforming the hull surface, to achieve a desired displacement and LCB. If the underlying geometry of the Form Topology curve network is to remain unchanged, two free form faces in the Form Topology Graph, from the entrance and the run respectively, are selected with which to modify the hydrostatic properties of the hull surface. Each face must expose a parameter with which the curves associated with the face may be deformed.

As the Form Topology curve network is to remain unchanged, the edges of the surface blended across the face will also remain unchanged. Consequently, the formulation of the surface blended across the face must be extended to introduce a deformation parameter creating either a bulge or flattening of the curve geometry associated with the face.
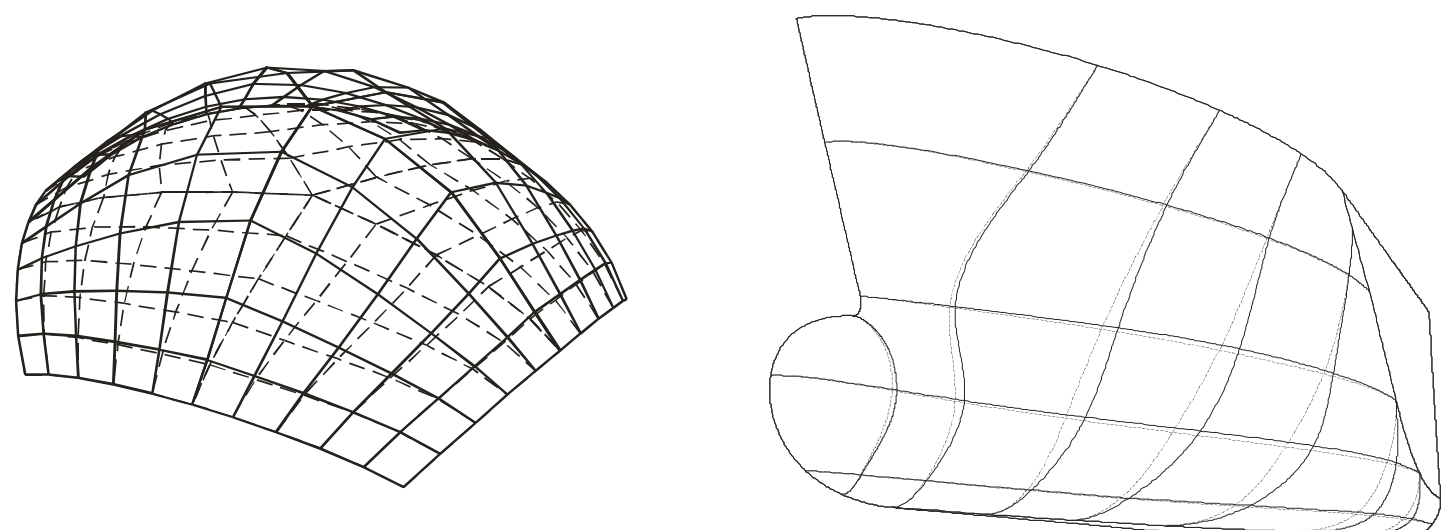

Fig.12: By introducing a bulging parameter into the surfaces blending across the faces of the form topology curve network, hydrostatic changes of the hull form can be introduced.

Gregory's multi sided Coons Patch can be extending to introduce the deformation parameter using existing principles in the formulation. In the technique, a local surface is generated between each pair of edges. Using a parameterisation known as a "footprint", the concept of which is best described in Gao (2005), a point is calculated on each local surface and combined using a weighted least squares to produce the final surface representation. Obviously, the surface can be linearly offset by adding a uniform vector to each point found local surface. However, a bulging effect can be achieved by using the weighted least squares combination and weighting parameterisation so that the offset applied in the centre of the surface is maximum and reduced to zero at the edges. The magnitude of this offset can be adjusted by the associated control parameter, with negative values causing the surface to flatten.

\subsection{Providing the Designer with Hydrostatic Transformation Capability}

In combination, the Form Topology curve network and the ability to modify the geometry of curves associated with freeform faces of the Form Topology graph provides the designer with a powerful capability for varying the hydrostatic properties of a hull form. However, it must be packaged in way which hides complexity and makes it accessible in during the design process. Reviewing the significant design parameters affecting hydrostatics, displacement (including $\mathrm{Cp}$ and $\mathrm{Cb}$ ), LCB, Length of PMB and position of PMB appear to be the principle quantities. Various combinations of these parameters can be used to invoke the hydrostatic changes previously described. A transformation schedule was developed which favoured changed to the PMB over modifications wholly within the entrance and run unless PMB was constrained. Some combinations of parameters require the PMB to be changed prior to modifying the freeform faces in the entrance and run. In situations where the PMB is partially constrained, i.e. only the extent of the PMB or the location of the PMB is constrained, a further iterative procedure is used to ensure that the amount of change applied to the freeform faces is balanced between each end of the vessel after the PMB has been updated. 
Finally, as only the extreme limits of Displacement and LCB are found when identifying the brackets, not all combinations of these values are possible resulting in a failure of the iteration process. Rather than flag an error to the designer, a process originally introduced into IntelliHull finds the closest solution to the desired hydrostatic properties. Consequently, the designer is able to understand more about the volumetric capabilities of the hull form.

\section{Designing the Hull Transformation Software Experience}

One of the striking observations about many commercial hull design tools, given the importance of hull transformations in the ship design process, is the poor experience it provides the designer. In the best of cases, transformations tools are provided separately to the definition experience. In the worst of cases, the designer may not have any control over how the results of the transformation are applied to the overall design or the surface may require a significant amount of preparation before reliable changes can be made. If there is any question over the robustness of the transformation tool, with the possibility that the surface definition could be ruined, the designer is unlikely to choose to use it.

To address issues found in commercial systems, the hull transformation experience in which the methods described previously are implemented was designed to be closely integrated into the surface definition process. It is provided as an extra tool that can be accessed at any point and aims to minimise the chance of breaking the designer's thought process or 'flow'. To provide a quality transformation experience, two key processes must be provided by the software.

\subsection{Management of the Surface Definition throughout the Transformation Process}

The designer may have spent many hours manipulating a hull surface but may then be faced with making a major change using a transformation. There are several reasons why a transformation could fail. The applied transformation could be inaccurate, it did not achieve the designer's expectations, the requested deformation could have been beyond what the surface definition could support and has undesirable features or due to poor software robustness. Consequently, the designer will need to return to the original definition as start again. The implementation of an Undo/Redo facility could achieve this, but it is not possible to implement any reviewing capabilities of the design changes as only one view of the surface definition can be seen at any time. To support the review of design changes and to ensure that the original definition is isolated from changes, a copy of the original surface definition is taken and each change produces a further copy in a stack. Each copy is associated with a description of the change. The designer can select different transformations in the stack providing the ability to go back or compare the definition with respect to the original definition. Once the designer has explored the options, a range of choices can be made. The changes can be applied to the original definition, to accept the change as a new surface definition or to abandon the process all together. Throughout all of these activities the original surface definition remains untouched until the designer commits or rejects the changes.

\subsection{Review of Transformation Changes}

One feature which is seldom provided when transforming a hull surface is the ability to compare it with the original design. Within the design environment it is possible to review geometrical features, but also check the hydrostatic properties and section area curve. When different copies of the design are held, it is possible to overlay the changes with respect to the original design both graphically and numerically. This provides the designer with confidence that the transformed provides the expected capabilities prior to accepting the change. However, it should be noted that care must be taken with the amount of information displayed on screen that the designer is not overloaded with information. Consequently, it is necessary to provide with the option of turning on the additional review features and begin from a minimal display state. 


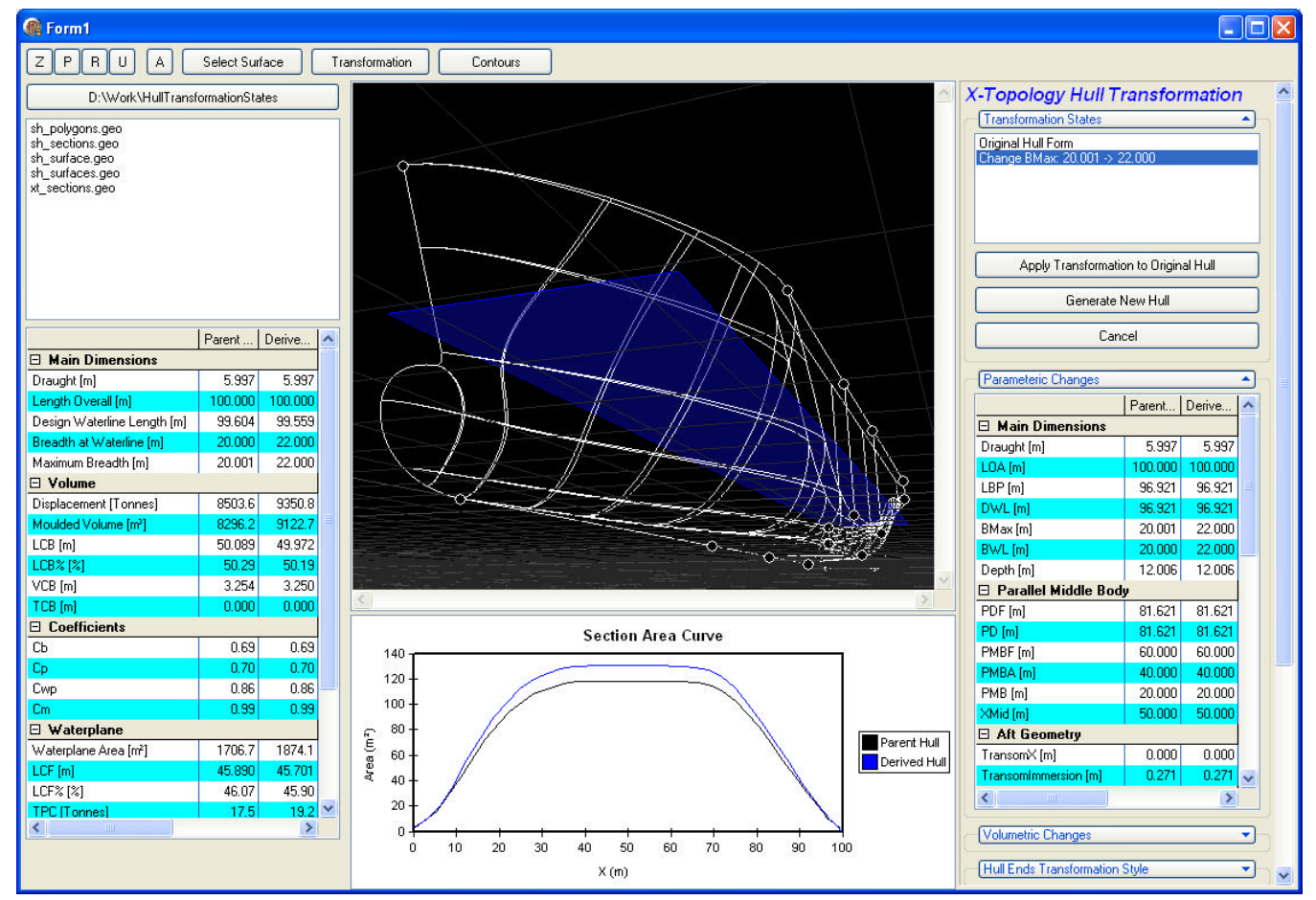

Fig 13: A prototype of the intended user interface illustrating the ability to compare the original design with respect to the changed design, both graphically and numerically.

\section{Observations}

Although development of this technique is incomplete it has shown itself to be robust from the beginning. Prior to the introduction of any parametric control, interactive manipulation was used to translate the position of vertices and, as this approach is often capable of making uncontrollably significant modifications, the method had to be robust enough to cope with this level of distortion. The main outstanding item of development remains to be the ability to reconnect curves when a change results in improper references in the main curve network. In isolation this would be a significant risk to the success of the work. However, techniques for automatic curve generation and connection are already available to the designer when creating a hull surface definition and the intension is to use a similar approach when transforming the curve definition.

In the author's experience, complex hull surface design operations often result in poor performance and limited robustness. However, this approach seems to go against this trend. The use of topology in any process increases its complexity significantly, certainly when comparing this approach to those introduced by Lackenby. In this particular case, topology is used to capture the features of the hull form allowing the geometrical transformations to be localised to specific regions of the surface definition and, as a consequence, the geometric operations are kept simple.

Due to the level of complexity encountered when dealing with topology, the author perceived that there was a significant risk that the approach would not succeed. This was the first opportunity to use Form Topology explicitly in combination with a surface definition technique based on curve networks since this was identified as the only way of extending IntelliHull. In IntelliHull, it was necessary to encode the Form Topology into the technique its self and the reliance on producing a single B-Spline limited the range of shapes. In this development, the Form Topology is identified from the curve network definition itself and from this the parametric transformations that are appropriate to the hull form are identified. At present, if a hull surface that did not conform to the tradition prismatic shape of a commercial hull form was used in this transformation it is unlikely that there would be many useful parameters available to the designer. However, it would still be possible to deform the hull surface using this technique using interactive manipulation. 
Although this approach departs significantly to those documented by Lackenby, his methods have proved invaluable when it came to devising routines to control the hydrostatic properties of the hull surface and in many ways could be described as a "first principles approach" to changing hydrostatic properties. It situations where it was possible transform the desired hydrostatic properties into prismatic coefficient for the entrance and run, it was possible to incorporate Lackenby's methods directly into the iteration routines. Consequently, Lackenby should be considered recommended reading for anyone attempting to develop routines to systematically change the hydrostatic properties of hull forms even if the techniques described do not apply directly.

\section{Concluding Remarks}

Although development on this hull transformation technique still continues, the following statements can be made about the principles used to develop this unique approach.

- Topology is a valuable tool that can be used to introduce alternative ways of manipulating hull surfaces beyond the direct manipulation of the basic surface definition.

- Form Topology can be used to identify specific parts of the hull form and subdivides it into a number of different regions allowing specific operations to be applied to the associated surface definition.

- It continues to be good advice to keep hull surface generation or manipulation rules as simple as possible to ensure robust performance and a wide range of applicability.

- Although rarely used for hull surface representation, Multi-sided surfaces have proved to be invaluable when trying to control surface shapes over the arbitrarily shaped regions within the Form Topology.

- The principles described by Lackenby in 1950 are still applicable to modern hull surface definition and remain valuable when devising routines for transforming the hull with respect to the hydrostatics properties.

\section{References}

BOLE, M. LEE, B. S. (2006), Integrating Parametric Hull Generation into Early Stage Design, Schiffstechnik Bd.53 - 2006/Ship Technology Research Vol. 53.

GAO, K. ROCKWOOD, A. (2005) Multi-sided Attribute Based Modelling, Mathematics of Surfaces, Lecture Notes in Computer Science 3604, pp. 219-232, 2005.

GREGORY, J.A. (1982), C1 rectangular and non-rectangular surface patches, Proceedings of Surfaces in Computer Aided Geometric Design, April 25-39, Oberwolfach, Germany, pp. 25-34.

LACKENBY, H (1950), On the Systematic Geometrical Variation of Ship Forms, Trans. INA, Vol. 92, pp. 289-315.

SÖDING, H., RABIEN, U. (1977), Hull Surface Design By Modifying An Existing Hull, Symposium on Computer-Aided Hull Surface Definition, Annapolis, Md.

VAN OVERVELD, C.W.A.M., VIAUD, M.L., (1996), Sticky Splines: Definition And Manipulation Of Spline Structures, ACM Transactions on Graphics (15), pp. 72-98. 